\title{
EDITORIAL
}

\section{Jahr KI bei Springer}

\author{
Josef Schneeberger
}

Online publiziert: 27. Januar 2011

(C) Springer-Verlag 2011

Liebe Leserinnen, liebe Leser!

Mit dem Jahreswechsel blickt die KI auf ein Jahr beim Springer-Verlag zurück. Für eine echte Bewertung ist es sicherlich zu früh, aber es ist ein guter Zeitpunkt für eine erste Zwischenbilanz. Die anvisierten Ziele - eine deutlichere Sichtbarkeit der KI und eine bessere Kombination internationaler und deutschsprachiger Beiträge - können sicher nicht in zwölf Monaten erreicht werden. Wie hat sich also die Zusammenarbeit mit Springer in 2010 entwickelt? Wenig überraschend ist die fachliche Kompetenz, mit der Springer die Hefte produziert und die einzelnen Artikel im Internet publiziert. Sie können alle Beiträge der KI vorab schon auf den Web-Seiten von Springer ${ }^{1}$ lesen. Deutlich stärker überrascht hat uns die freundschaftliche Unterstützung, die den KI Herausgebern von den Mitarbeitern des SpringerVerlags entgegengebracht wurde. Unser Dank gilt hier vor allem Frau Glaunsinger, Frau Uhl und Herrn Engesser! Sie haben viel Verständnis für unsere spezifischen Wünsche aufgebracht und sich auch auf ungewohnte, neue Arbeitsweisen eingelassen.

Da KI-Hefte mit einem Vorlauf von 1-2 Jahren zusammengestellt werden, hat in 2010 eine schrittweise Verlagerung der Produktion der KI-Hefte stattgefunden. Wenn Sie heute einen Fachbeitrag zur KI einreichen (wozu ich Sie herzlich einlade!), dann wird der gesamte Prozess - von der Anmeldung über die Begutachtung bis hin zum Druck - im Springer-System abgewickelt. Das vorliegende Heft ist ein typisches Beispiel für die Umstellung: Eingang und Begutachtung der Beträge wurden vom Gastherausgeber Mi-

${ }^{1}$ Siehe: http://www.springer.com/computer/ai/journal/13218.

J. Schneeberger $(\varangle)$

FB Wirtschaftsinformatik, Hochschule Deggendorf, Edlmairstr. 6

+ 8, 94469 Deggendorf, Deutschland

e-mail: josef.schneeberger@fh-deggendorf.de chael Thielscher selbst organisiert, während Produktion und Druck im Springer-System abliefen.

Im Jahr 2010 hat sich auch der Kreis der Herausgeber deutlich verändert. Andreas Günter, der die KI viele Jahre geprägt hat, ist als Hauptherausgeber ausgeschieden und hat seine Aufgaben an Klaus-Dieter Althoff übergeben. Auf der letzten Sitzung des Fachbereichs im September wurden auch zwei neue Redakteure der KI bestätigt, die ich hiermit herzlich bei der KI begrüßen will: Simone Fintrop ist Mitarbeiterin an der Universität Bonn und sie verstärkt die Redaktion mit ihrer Kompetenz im Bereich Intelligente Bildverarbeitung und Bildverstehen. Der zweite neue Herausgeber ist Daniel Sonntag, ein Mitarbeiter am DFKI in Saarbrücken. Seine Schwerpunkte liegen unter anderem bei Applikationen des Semantic Web und bei Dialogsystemen. Beide Redakteure werden sich in Kürze hier mit einem eigenen Editorial vorstellen. Ich selbst darf mich nach 12 Jahren der Mitarbeit an der KI mit diesem Editorial bei Ihnen verabschieden. Es hat viel Spaß gemacht, die Entwicklung der Zeitschrift über die Jahre zu verfolgen und zu fördern.

Im vorliegenden Heft hat Michael Thielscher spannende Beiträge zum Thema „General Game Playing“ zusammengestellt. Mit der internationalen Ausrichtung kann dieses Heft ein Prototyp einer erneuerten KI sein. General Game Playing hat sich in den letzten Jahren zu einem wichtigen Gebiet der KI entwickelt. Es verbindet exemplarisch klassische theoretische Ansätze aus Logik und Wissensrepräsentation mit Konzepten für die effiziente Implementierung von Anwendungen. Viele betrachten dieses Gebiet auch als eine neue Klammer zur Kerninformatik, da klassische Informatiktheorien hier eine wichtige Rolle spielen. Das Heft gibt einen guten Überblick über das (noch junge) Gebiet und einen Eindruck über den Stand der Arbeiten.

Viel Spaß beim Lesen wünscht Ihnen

Josef Schneeberger 


\section{Themenvorschau}

\section{Schwerpunkt - Off-road Robotik}

Als Off-road Roboter werden Systeme bezeichnet, die autonom bzw. teilautonom in rauem, natürlichem und unebenen Gelände agieren. Im Gegensatz zur Forschung im Bereich der kognitiven Automobile zeichnet sich die Off-road Robotik durch die Unstrukturiertheit der Umwelt, die Variabilität der Umgebungsbedingungen, die zu berücksichtigende Dreidimensionalität und die hohe Hindernisdichte aus. Die Unstrukturiertheit und die Variabilität der Umwelt erschweren zum einen die Erkennung der Umweltgegebenheiten und zum anderen die Navigation solcher Systeme.

Im Rahmen des Schwerpunkts Off-road Robotik werden Beiträge zu folgende Bereichen vorgestellt:

- Umweltrepräsentation und Karten

- Situationserkennung und die Geländeeinschätzung

- Lokale und globale Navigationsverfahren

- Anwendungsprojekte

Interessierte Autorinnen und Autoren werden gebeten, sich an den Gastherausgeber zu wenden.

\section{Prof. Dr. Karsten Berns}

AG Robotersysteme

Fachbereich Informatik

Technische Universität Kaiserslautern

Postfach 3049

67653 Kaiserslautern

berns@informatik.uni-kl.de

\section{Schwerpunkt - Emotion and Computing}

How does emotion and computing work together? Can a computer be emotional? Can a computer system recognize, understand and react on human emotions? In recent years computer science research has dealt with these questions in numerous projects. Several approaches have been made concerning emotion recognition, emotion modeling, generation of emotional user interfaces and dialogue systems as well as anthropomorphic communication agents. Regarding human behaviour, emotions play an essential role in decision making, as well as in perception and learning. Modern media take an increasing role regarding human social interaction. Human computer interfaces integrate the recognition and synthesis of nonverbal parts of communication.

This special issue of KI focusses on the role of artificial intelligence in emotional computer systems. This inclu- des computational models of emotion, their applications and the methods of artificial intelligence applied. More detailed information can be found on http://www.emotion-andcomputing.de/. Interested authors are asked to contact the guest editor as soon as possible:

\section{Prof. Dr. Dirk Reichardt}

Prorektor und Dekan Fakultät Technik

Professor für Informatik

Duale Hochschule Baden-Württemberg Stuttgart

Jägerstraße 56

70174 Stuttgart

reichardt@dhbw-stuttgart.de

\section{Schwerpunkt - 25 Jahre KI und KI-Zeitschrift}

Seit 1987 erscheint die Zeitschrift „Künstliche Intelligenz“ in einem Verlag, der Vorgänger „KI-Rundbrief“ wurde als Manuskript von Universitäten bzw. Forschungseinrichtungen kopiert und verteilt. In diesem ,Jubiläumsheft“ wird unter verschiedenen Aspekten auf diese 25 Jahre KI und KIZeitschrift zurückgeblickt.

Darüber hinaus ist geplant, auch die nähere und fernere Zukunft der KI und der Zeitschrift zu beleuchten.

Interessierte Autorinnen und Autoren werden gebeten, sich möglichst bald an den Gastherausgeber zu wenden. Es sind zur Vergangenheit und Zukunft der KI und ihrer Zeitschrift Beiträge aller Art erwünscht!

\author{
Dr. Andreas Günter \\ HITeC e.V./Uni Hamburg \\ Vogt-Kölln-Str. 30 \\ 22527 Hamburg, Deutschland \\ Tel: 040/42883-2456 \\ guenter@informatik.uni-hamburg.de
}

\section{Schwerpunkt - KI Sprachen (AI Languages)}

In the past, mainly Prolog and Lisp were considered as the most important representatives of artificial intelligence programming languages. However, in the meantime, the range of such languages has been extended by the development of new areas of interest within AI. Existing languages have been extended, e.g. by constraints, and there are developments of multi-paradigm languages which support more than one programming paradigm and style with the aim of an efficient modelling and problem solving. But, furthermore, there are new directions of research and application, like languages of the Semantic Web, languages for planning and agent-oriented programming. 
This special issue includes technical papers, reports on current research projects, interviews, summaries of doctoral theses, book reviews and others with focus on current developments and perspectives of languages in the area of AI. Topics of interest include but are not limited to:

- Classical and new paradigms of AI programming

- Functional-logic, constraint-based, and further multiparadigm languages

- Inductive programming

- Languages of the Semantic Web

- Domain specific languages which support programming of AI-specific applications, e.g. planning applications and agent-oriented programming
Interested authors should contact the guest editor as soon as possible:

\section{Prof. Dr. Petra Hofstedt}

Brandenburgische Technische Universität Cottbus Fakultät 1, Institut für Informatik

Lehrstuhl Programmiersprachen und Compilerbau

Postfach 101344

03013 Cottbus

hofstedt@informatik.tu-cottbus.de 